\title{
Reward-Related Cortical Inputs Define a Large Striatal Region in Primates That Interface with Associative Cortical Connections, Providing a Substrate for Incentive-Based Learning
}

\author{
Suzanne N. Haber, ${ }^{1}$ Ki-Sok Kim, ${ }^{2}$ Philippe Mailly, ${ }^{3}$ and Roberta Calzavara ${ }^{1}$ \\ ${ }^{1}$ Department of Pharmacology and Physiology, University of Rochester School of Medicine, Rochester, New York 14642, ${ }^{2}$ Department of Public Health, \\ Keimyung University, Taegue 704-701, Korea, and ${ }^{3}$ Neurobiologie des Signaux Intercellulaires, Centre National de la Recherche Scientifique, Universite \\ Pierre et Marie Curie, 75252 Paris, France
}

\begin{abstract}
The anterior cingulate and orbital cortices and the ventral striatum process different aspects of reward evaluation, whereas the dorsolateral prefrontal cortex and the dorsal striatum are involved in cognitive function. Collectively, these areas are critical to decision making. We mapped the striatal area that receives information about reward evaluation. We also explored the extent to which terminals from reward-related cortical areas converge in the striatum with those from cognitive regions. Using three-dimensional-rendered reconstructions of corticostriatal projection fields along with two-dimensional chartings, we demonstrate the reward and cognitive territories in the primate striatum and show the convergence between these cortical inputs. The results show two labeling patterns: a focal projection field that consists of densely distributed terminal patches, and a diffuse projection consisting of clusters of fibers, extending throughout a wide area of the striatum. Together, these projection fields demonstrate a remarkably large, rostral, reward-related striatal territory that reaches into the dorsal striatum. Fibers from different reward-processing and cognitive cortical areas occupy both separate and converging territories. Furthermore, the diffuse projection may serve a separate integrative function by broadly disseminating general cortical activity. These findings show that the rostral striatum is in a unique position to mediate different aspects of incentive learning. Furthermore, areas of convergence may be particularly sensitive to dopamine modulation during decision making and habit formation.
\end{abstract}

Key words: decision making; incentive learning; dorsolateral prefrontal cortex; orbital cortex; cingulate cortex; ventral striatum; dopamine

\section{Introduction}

A key component to good decision making is the ability to accurately evaluate elements of outcome, including reward value, predictability, and risk. Different prefrontal cortical areas and corresponding striatal regions are involved in various aspects of reward evaluation and incentive-based learning (Elliott et al., 2000b, 2003; Schultz et al., 2000; Knutson et al., 2001; Pagnoni et al., 2002) and are associated with pathological risk taking and addictive behaviors (Kuhnen and Knutson, 2005; Volkow et al., 2005). The anterior cingulate cortex (ACC) and orbital cortex (OFC) mediate the different aspects of reward-based behaviors, error prediction, and the choice between short- and long-term gains. The dorsolateral prefrontal cortex (DLPFC) is engaged when working memory is required for monitoring behavior (Goldman-Rakic et al., 1990; Elliott et al., 2000a; Hadland et al., 2003; Walton et al., 2004; Fellows and Farah, 2005). The ACC/

Received Jan. 20, 2006; revised July 10, 2006; accepted July 11, 2006

This work was supported by National Institutes of Health Grant MH45573.

Correspondence should be addressed to Dr. Suzanne N. Haber, Department of Pharmacology and Physiology, University of Rochester School of Medicine, 601 Elmwood Avenue, Rochester, NY 14642. E-mail: suzanne_haber@urmc.rochester.edu.

D0I:10.1523/JNEUROSCI.0271-06.2006

Copyright $\odot 2006$ Society for Neuroscience $\quad$ 0270-6474/06/268368-09\$15.00/0
OFC and DLPFC are connected to the nucleus accumbens (or ventral striatum) and the caudate nucleus, respectively (Yeterian and Van Hoesen, 1978; Selemon and Goldman-Rakic, 1985; Haber et al., 1995; Ferry et al., 2000). However, these connectivity studies have emphasized a directed topography of connections, supporting a model of segregated and parallel corticobasal ganglia circuits (Alexander et al., 1990). Nonetheless, rewardresponsive activation is not restricted to the ventral striatum but is found throughout the striatum (Hassani et al., 2001; Takikawa et al., 2002; Tanaka et al., 2004). Because the reward signal, critical to the striatal role in learning, is generated from the midbrain dopamine cells (Schultz, 2002) and conveyed to each of the separate and parallel cortical pathways within the striatum, little attention has been given to a possible role for the direct ACC/ OFC striatal inputs for incentive learning outside of the relatively restricted ventral striatal region. Our experiments were designed to (1) determine the extent of the reward-related striatum based on ACC/OFC cortical inputs and (2) determine whether there is convergence between these inputs and with those from the DLPFC. Such convergence would influence reward-based learning and may be particularly sensitive to the dopamine signal (Haber et al., 2000; Everitt and Robbins, 2005).

Recent human imaging studies have divided the prefrontal 

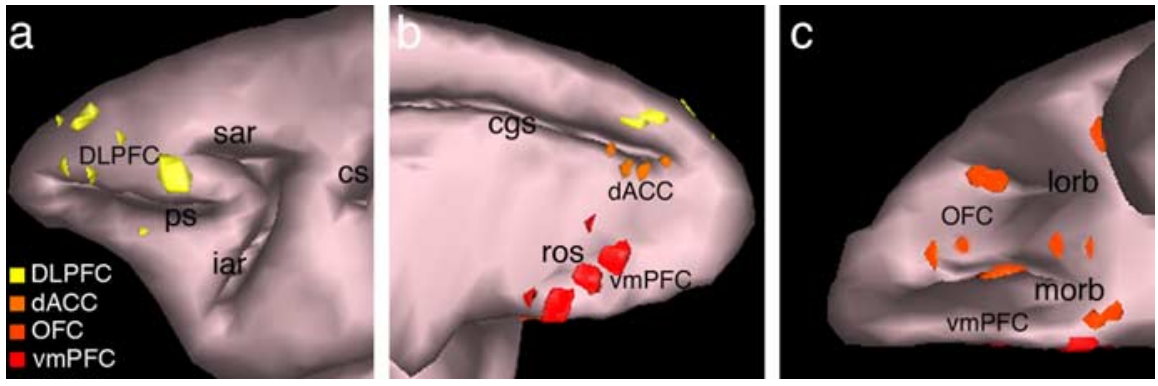

Figure 1. Injection site placements. $\boldsymbol{a}$, Dorsolateral view of the brain showing the DLPFC injection sites in yellow; $\boldsymbol{b}$, medial view showing the $\mathrm{dACC}$ and vmPFC injection sites in orange and red, respectively; c, ventral view showing the OFC injection sites in dark orange. Note that the injection sites cover a relatively small, restricted part of each functional region. cgs, Cingulate sulcus; cs, central sulcus; iar, inferior arcuate sulcus; lorb, lateral orbital sulcus; morb, medial orbital sulcus; ps, principal sulcus; ros, rostral sulcus; sar, superior arcuate sulcus.

cortex into the dorsal anterior cingulate cortex (dACC), the ventral, medial prefrontal cortex (vmPFC), OFC, and DLPFC based on specific roles for mediating different aspects of error prediction and decision making (Elliott et al., 2000a,b; Walton et al., 2004; Fellows and Farah, 2005). Using these divisions, we charted the vmPFC, dACC, OFC, and DLPFC corticostriatal terminals in primate and developed three-dimensional (3-D) reconstructions of their projection fields. The results show two patterns: a focal projection field forming relatively confined, dense terminal patches (Graybiel and Penney, 1999), and a diffuse projection consisting of fiber clusters extending more widely throughout the striatum. Together, the projections from vmPFC/dACC/OFC demonstrate an extensive reward-related striatum. Of particular importance, the results also show a complex interweaving and convergence between these cortical inputs, providing an anatomical substrate for modulation between circuits to affect incentive learning and habit formation.

\section{Materials and Methods}

To examine the organization of OFC and ACC corticostriatal projections, we injected anterograde tracers into the anterior cingulate cortex (areas 24, 32, and 25), orbital cortex (areas, 11, 13, 14, and 12), and dorsolateral prefrontal cortex (areas 9 and 46). Corticocortical labeling was used to verify the specificity of the injection sites. We charted the entire projection field throughout the striatum for each case. In addition to the traditional charting of individual fibers, we outlined the dense (or focal) projection fields for each case to create 3-D maps of the fields. These 3-D maps were then compiled to delineate the entire striatal region that receives the primary input from the ACC and OFC. The projection fields from the ACC and OFC were joined to define the volume of striatum influenced by reward-processing regions of prefrontal cortex. To verify convergent projection fields, four monkeys had multiple injections placed in different prefrontal cortex areas. In addition, the charted fibers outside the focal projection field (referred to as diffuse projections) were compiled from each cortical area into one map to illustrate the extent of its entire terminal region. These were then joined with the charts illustrating the focal projection fields from the different prefrontal cortex areas to determine the extent of possible interaction between different prefrontal cortex regions.

Surgery and tissue preparation. Twenty-two adult macaque monkeys (20 Macaca nemestrina and 2 Macaca fascicularis) were used for the tracing studies. Magnetic resonance imaging (MRI) images from two additional $M$. fascicularis were used to compare the average striatal volume of the two species (see below, Data analysis). All experiments were conducted in accordance with the Guide for the Care and Use of Laboratory Animals (National Research Council, 1996) and were approved by The University Committee on Animal Resources. Monkeys were tranquilized by intramuscular injection of ketamine $(10 \mathrm{mg} / \mathrm{kg})$. A surgical plane of anesthesia was maintained by intravenous injection of pentobarbital (initial dose of $20 \mathrm{mg}$ / $\mathrm{kg}$, i.v., and maintained as needed). Temperature, heart rate, and respiration were monitored throughout the surgery. Monkeys were placed in a David Kopf Instruments (Tujunga, CA) stereotaxic, a midline scalp incision was made, and the muscle and fascia were displaced laterally to expose the skull. A craniotomy $(\sim 2-3$ $\mathrm{cm}^{2}$ ) was made over the region of interest, and small dural incisions were made only at recording or injection sites. To guide deep cortical injections, serial electrode penetrations were made to locate the anterior commissure as described previously (Haber et al., 1993). The absence of cellular activity signaled the area of fiber tracts, i.e., the corpus callosum, the internal capsule, and the anterior commissure. We calculated the anterior/posterior position of various orbital regions based on the location of the anterior commissure. Additional recordings were performed to determine the depth of the injection sites. Accurate placement of tracer injections was achieved by careful alignment of the injection cannulas with the electrode. In several animals, we obtained magnetic resonance images to guide our injection sites. The dorsolateral injections sites were determined by visual inspection of frontal cortical gyri, indicating general frontal cortical areas.

Monkeys received an injection of one or more of the following anterograde/bidirectional tracers: Lucifer yellow (LY), Fluororuby (FR), or fluorescein (FS) conjugated to dextran amine $[40-50 \mathrm{nl}, 10 \%$ in $0.1 \mathrm{M}$ phosphate buffer (PB), pH 7.4; Invitrogen, Carlsbad, CA] Phaseolus vulgaris leucoagglutinin (PHA-L) (50 nl, 2.5\%; Vector Laboratories, Burlingame, CA), or tritiated amino acids (AA) $\left(100 \mathrm{nl}, 1: 1\right.$ solution of $\left[{ }^{3} \mathrm{H}\right]$ leucine and $\left[{ }^{3} \mathrm{H}\right]$-proline in $\mathrm{dH}_{2} \mathrm{O}, 200 \mathrm{mCi} / \mathrm{ml}$; NEN, Boston, $\mathrm{MA}$ ). Tracers were pressure injected over $10 \mathrm{~min}$ using a $0.5 \mu$ l Hamilton syringe. After each injection, the syringe remained in situ for 20-30 min. Twelve to $14 \mathrm{~d}$ after the operation, monkeys were again deeply anesthetized and perfused with saline, followed by a $4 \%$ paraformaldehyde $/ 1.5 \%$ sucrose solution in $0.1 \mathrm{~m}$ phosphate buffer, $\mathrm{pH}$ 7.4. Brains were postfixed overnight and cryoprotected in increasing gradients of sucrose $(10,20$, and $30 \%$ ). Serial sections of $50 \mu \mathrm{m}$ were cut on a freezing microtome into $0.1 \mathrm{M}$ phosphate buffer or cryoprotectant solution as described previously (Haber et al., 2000).

Immunocytochemistry was performed on free-floating sections (one in eight for each tracer) to visualize LY, FR, FS, and PHA-L tracers. Before incubation in primary antisera, tissue was treated with $10 \%$ methanol and $3 \%$ hydrogen peroxide in $0.1 \mathrm{M} \mathrm{PB}$ to inhibit endogenous peroxidase activity and rinsed $1-2 \mathrm{~h}$ in PB with $0.3 \%$ Triton X-100 (TX) (Sigma, St. Louis, MO). Sections were preincubated in $10 \%$ normal goat serum (NGS) and $0.3 \% \mathrm{TX}$ in PB for $30 \mathrm{~min}$. Tissue was placed in the primary anti-LY (1:3000 dilution; Invitrogen), anti-FS (1:1000; Invitrogen), anti-FR (1:1000; Invitrogen), or anti-PHA-L (1:500; EY Laboratories, San Mateo, CA) in $10 \%$ NGS and $0.3 \%$ TX in PB for 4 nights at $4^{\circ} \mathrm{C}$. After extensive rinsing, the tissue was incubated in biotinylated secondary antibody, followed by incubation with the avidin-biotin complex solution (Vectastain ABC kit; Vector Laboratories). Immunoreactivity was visualized using standard DAB procedures. Staining was intensified by incubating the tissue for $5-15 \mathrm{~min}$ in a solution of $0.05 \% 3,3^{\prime}$ diaminobenzidine tetra-hydrochloride, $0.025 \%$ cobalt chloride, $0.02 \%$ nickel ammonium sulfate, and $0.01 \% \mathrm{H}_{2} \mathrm{O}_{2}$ to yield a black reaction product. Sections were mounted onto gel-coated slides, dehydrated, defatted in xylenes, and coverslipped with Permount. In cases in which more than one tracer was injected into a single animal, adjacent sections were processed for each antibody reaction.

Data analysis. Cortical injections with contamination or weak labeling were eliminated from the analysis. Contamination refers to all injections in which the tracer was not limited to a single cortical region but had leaked into an adjacent area or into the underlying white matter. Weak labeling refers to relatively few labeled fibers in the striatum labeling, 

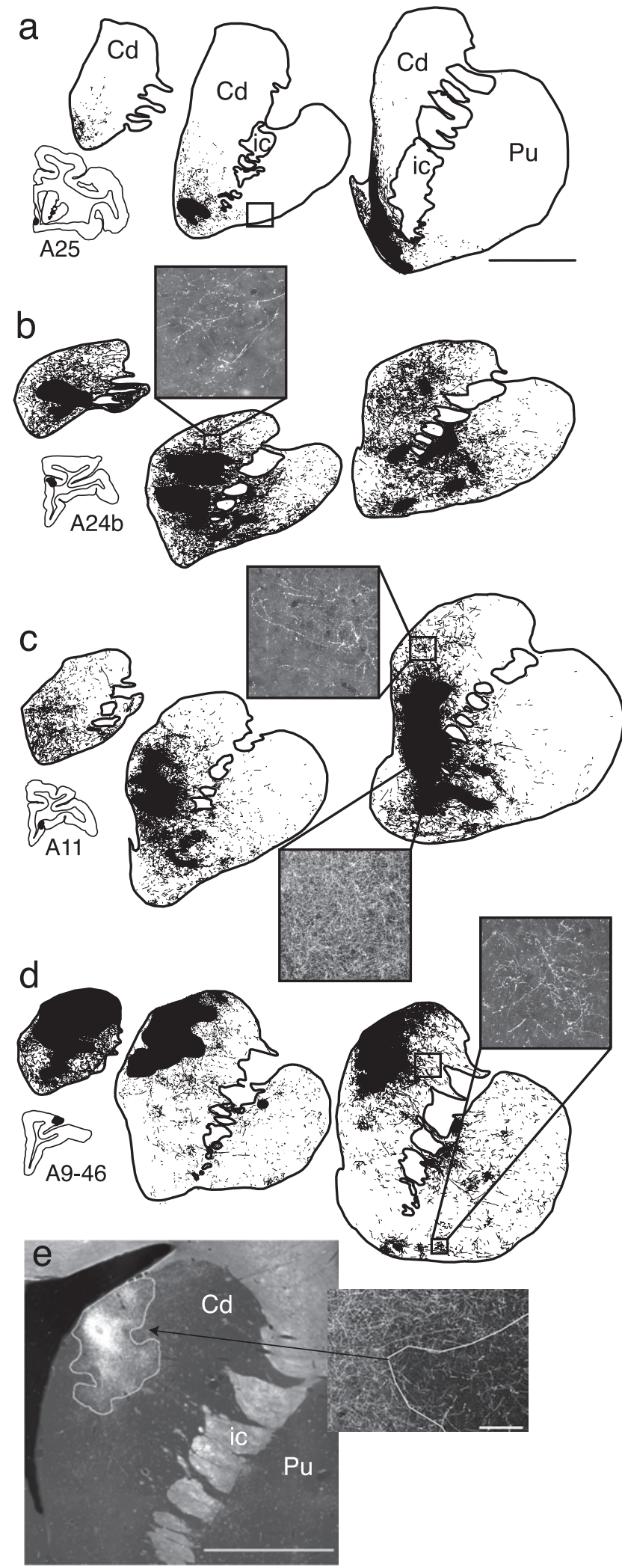

Figure 2. Schematic chartings and microphotographs of labeled fibers after injections into different prefrontal regions. $\boldsymbol{a}, \mathrm{vmPFC}$ injection site (area 25); $\boldsymbol{b}$, dACC injection site (area 24b); c, OFC injection site (area 11); $\boldsymbol{d}$, DLPFC injection site (area 9/46). The focal projection fields (black area) and the diffuse projections are shown in charts at three anteroposterior levels, rostral to the anterior commissure. $e$ illustrates microphotographs at low and high magnification $(1.6 \times$, scale bar, $5 \mathrm{~mm} ; 10 \times$, scale bar, $100 \mu \mathrm{m})$ of the dense terminal field charted in $\boldsymbol{d}$ (the most caudal section) with the border of the focal projection. Cd, Caudate nucleus; ic, internal capsule; Pu, putamen nucleus. typically the result of the injection site centered in superficial cortical layers. All thin, labeled fibers containing boutons were charted. Thick fibers without clear terminal boutons were assumed to be passing fibers and were not included. Fiber distributions for each case were charted throughout the rostrocaudal striatum. We divided the ACC/OFC into the following: the dACC, areas $24 \mathrm{~b}$ and dorsal 32; the vmPFC, areas 25 and $14 \mathrm{~m}$; and the OFC, areas 11,13 , and 12 . There were four injections into different regions of the dACC, six into the vmPFC, nine into different regions of OFC, and seven into the DLPFC (Fig. 1). Four animals received multiple injections into different regions of the prefrontal cortex. The general corticocortical labeling in each case was consistent with expected results from on target injections (Barbas and Pandya, 1989; Carmichael and Price, 1996). For the anatomical boundaries of ACC, OFC, and DLPFC, we used the atlas by Paxinos et al. (2000) in conjunction with detailed anatomical descriptions (Barbas and Pandya, 1989).

3-D reconstructions. 3-D reconstructions of focal projection fields in the striatum were developed to (1) address how each focal projection lies within the striatum in dorsal/ventromedial/lateroanterior/posterior space, and (2) develop a 3-D global model to compare multiple focal projections from different cortical regions. Previous studies have shown (at low magnification) relatively large, dense patches of corticostriatal projections (Yeterian and Van Hoesen, 1978; Selemon and GoldmanRakic, 1985; Parthasarathy et al., 1992). We used the ability to visualize these dense projections at low magnification to create the $3-D$ renderings for the focal projections. These areas were determined using a $1.6 \mathrm{objec}-$ tive and the Neurolucida software (MicroBrightfield, Colchester, VT) to directly outline the region. Adjacent patches of dense fibers visible at $1.6 \times$ that were clearly surrounded by a less dense area, but visualized at $4 \times$, were connected and considered as one object. Isolated patches were treated as individual objects. Boundaries for each focal projection were checked for accuracy with the chartings that were performed at higher magnification $(10 \times)$ (see Fig. 2e). For each case, a stack of 2-D coronal sections was created from its Neurolucida chartings and Nissl images. This stack was imported into IMOD, a 3-D rendering program (Boulder Laboratory for 3D Electron Microscopy of Cells, University of Colorado, Boulder, CO) (Kremer et al., 1996), and a 3-D reconstruction that contained the focal projection field was created for each case separately. To merge several cases together, we developed a reference model of the striatum from one animal. This model was created by sampling one in eight sections (at $400 \mu \mathrm{m}$ intervals) throughout the striatum, using alternate sections stained with acetylcholinesterase, Nissl, and enkephalin immunoreactivity. Data from each case was then transposed into the reference striatum using landmarks of key internal structures surrounding the striatum. After the transposition of focal projections from each case, every contour placed in the reference model was checked with the original for medial/lateral, dorsal/ventral, and anterior/posterior placement and relative size. This ensured that the focal projection field from each case was accurately placed with respect to its position and the proportion of the striatum it occupied. Thus, a 3-D rendering was created first for each single case and then for the combinations of cases. To determine convergence between focal projection fields, the model was "sectioned" throughout its rostrocaudal extent (see Fig. 3). Final verification of convergence was demonstrated with dual injections into a single animal (see Fig. 4). Using MRI data obtained for eight monkeys (four M. nemestrina and four M. fascicularis), we calculated the volume of the striatum. We found that the volume within each species was relatively similar, but, consistent with a smaller overall brain, the striatum in the $M$. fascicularis is somewhat smaller compared with the M. nemestrina ( $M$. nemestrina, $1285 \pm 42 \mathrm{~mm}^{3}$; M. fascicularis, $943 \pm 41 \mathrm{~mm}^{3}$; mean \pm SE).

\section{Results}

\section{General terminal distribution}

Figure 2 illustrates the complete projection from a representative tracer injection into the different prefrontal regions. There were two labeling patterns in the striatum, a focal projection field and a diffuse one (Fig. 2). The focal projection field consisted of dense clusters of terminals forming the well known dense patches. These could be visualized with relatively low magnification 

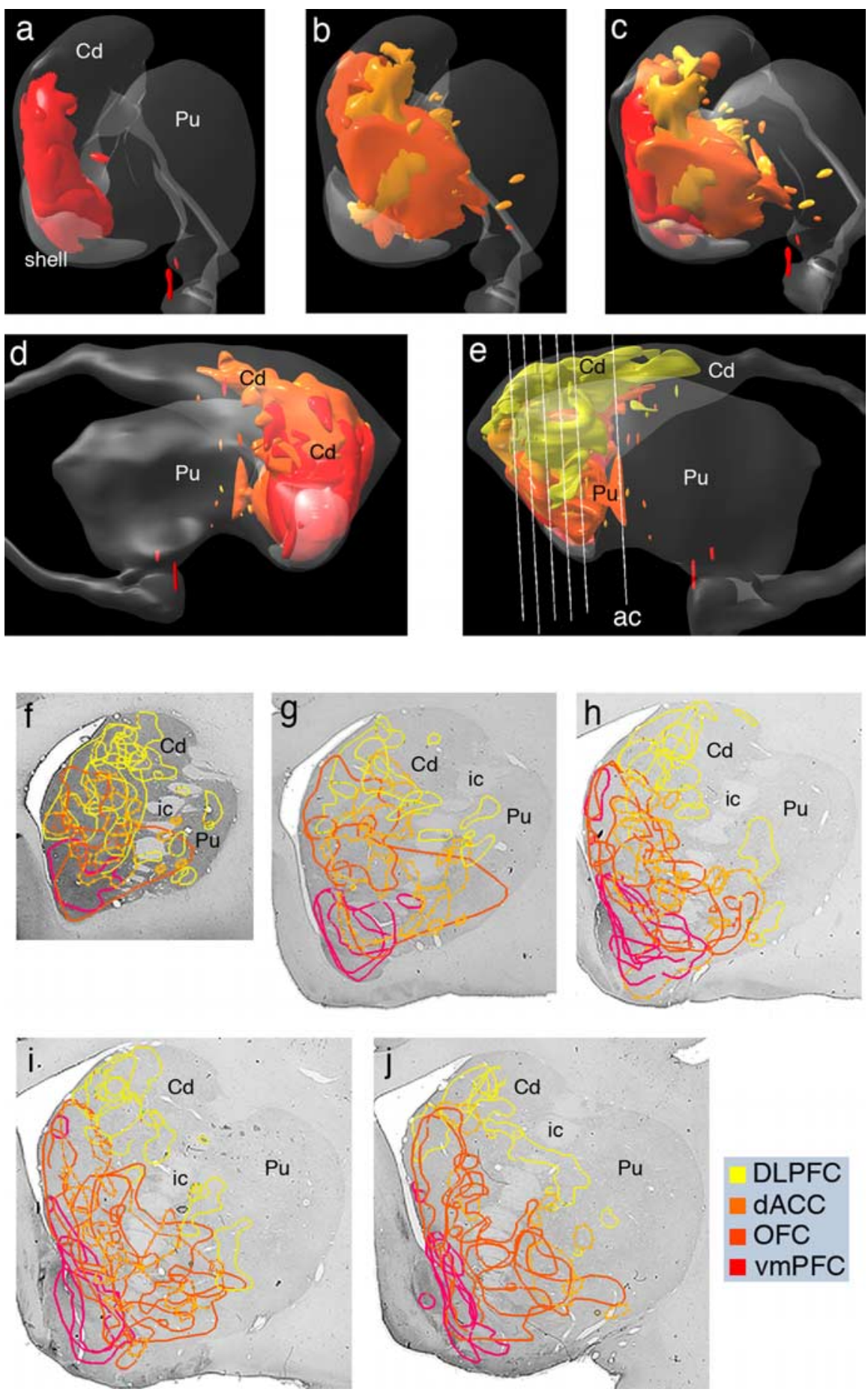

Figure 3. 3-D renderings of the combined projection fields from the PFC regions. $\boldsymbol{a}-\boldsymbol{c}$, Frontal view of the striatum showing inputs from the $\operatorname{vmPFC}(\boldsymbol{a})$, from both $\operatorname{dACC}$ and $\operatorname{OFC}(\boldsymbol{b})$, and from all PFC areas, including $\operatorname{DLPFC}(\boldsymbol{c}) ; \boldsymbol{d}, \boldsymbol{e}$, medial $(\boldsymbol{d})$ and lateral $(\boldsymbol{e})$ views showing all PFC inputs. White lines in $\boldsymbol{e}$ indicate the level of sections illustrated in $\boldsymbol{f}-\boldsymbol{j}$. $\boldsymbol{f} \boldsymbol{j}$, Coronal slices through the 3-D model with their corresponding Nissl section: red/purple, Inputs from vmPFC; dark orange, inputs from OFC; light orange, inputs from dACC; yellow, inputs from DLPFC. ac, Anterior commissure; Cd, caudate nucleus; ic, internal capsule; Pu, putamen nucleus.

$(1.6 \times)$. The general topography of the focal projections from vmPFC/dACC/OFC terminated in the rostral, medial, and ventral parts of the striatum, and the DLPFC projected primarily in the head of the caudate and part of the rostral putamen as reported previously (Selemon and Goldman-Rakic, 1985; Kunishio and Haber, 1994; Haber et al., 1995; Ferry et al., 2000). The diffuse projections consisted of clusters of terminal fibers that were

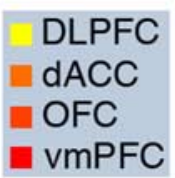

widely distributed throughout the striatum, both expanding the borders of the focal terminal fields and also extending widely throughout other regions of the striatum.

\section{The focal projection fields}

The focal projection field from the vmPFC was the most limited and was concentrated at the level of the nucleus accumbens, within and dorsal to the shell (Fig. 2a). These injections resulted in corticocortical labeling in other medial subgenual regions and in areas $24 \mathrm{a}$ and 12 , with few or no fibers terminating in areas $24 \mathrm{~b}$ and $24 \mathrm{c}$ or in areas 9 and 46. Projections from the dACC extended from the rostral pole of the caudate nucleus to the anterior commissure and were primarily located dorsal to the nucleus accumbens in both the central caudate nucleus and putamen but were not adjacent to the ventricle (Fig. 2b). They primarily avoided the shell region, although there was one dense terminal patch within the shell. Injections into different rostrocaudal regions of the dACC resulted in dense corticocortical labeling in area 9 and throughout area 24c (motor cingulate cortex). The OFC also projected to both the caudate nucleus and putamen but terminated somewhat medial and caudal to those from the dACC (Fig. 2c). Projections terminated primarily in a strip along the centromedial caudate nucleus extending ventral and lateral into the putamen (Fig. 2b). Despite the extensive overlap between the projection fields from each OFC injection site, there were some small topographic shifts depending on the medial/lateral position of the injection within the OFC. Injections placed into the OFC resulted in cortical labeling that was primarily located in other orbital regions, lateral to the gyrus rectus and in areas 9 and 46. Projections from the DLPFC were found from the beginning of the rostral pole and continued for $14.5 \mathrm{~mm}$, through much of the body of the caudate nucleus and medial putamen (Fig. $2 d$ ). Cortical fibers from these injection sites terminated primarily in the AACC (areas $24 \mathrm{~b}$ and $24 \mathrm{c}$ ), lateral OFC area 8 , and rostral premotor regions. Injection sites centered in area 46 occupied a similar rostrocaudal region as that of area 9, except these terminals were located more laterally and densely in the putamen.

The relationships between focal terminal fields from each cortical region are demonstrated using the collective $3-\mathrm{D}$ reconstructions from three different views: coronal, medial, and lateral (Fig. $3 a-e$ ). To view the detailed relationship between the different focal projection fields, the model was sectioned at five different levels (Fig. $3 f-j$ ). The vmPFC had the most limited projec- 

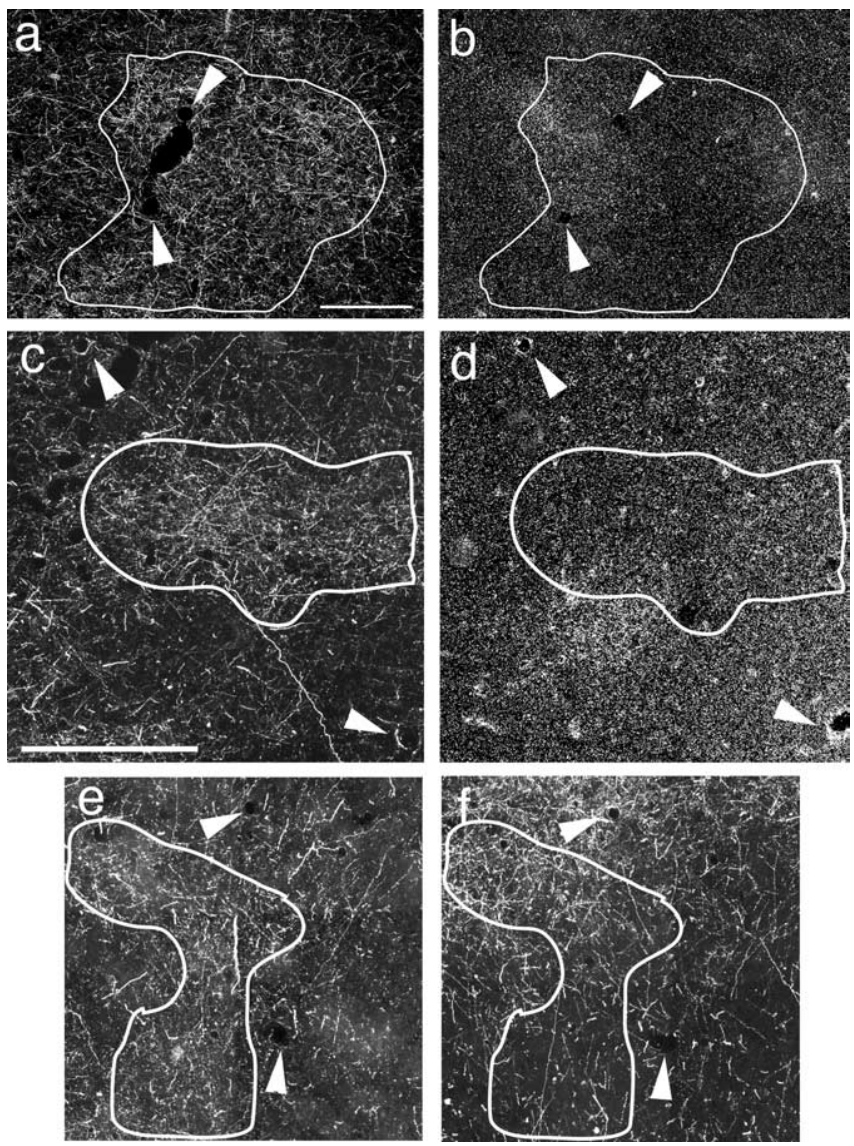

Figure 4. Photomicrographs of adjacent coronal sections of the striatum showing the labeled terminals after dual tracer injections into different PFC areas. $\boldsymbol{a}, \boldsymbol{b}$, Labeled striatal fibers after injections into dACC and OFC (area 24b, $\boldsymbol{a}$; area 13, b). Scale bar, $500 \mu \mathrm{m}$. c, d, Labeled striatal fibers after injections into vmPFC and OFC (area 14, c; area 13, d). Scale bar, $500 \mu \mathrm{m} . \boldsymbol{e}$, $\boldsymbol{f}$, Labeled striatal fibers after injections into dACC and OFC (area 32, $\boldsymbol{e}$; area 11, $\boldsymbol{f}$ ). The scale bar is shown in $\boldsymbol{c}$. White outline on $\boldsymbol{a}, \boldsymbol{c}$, and $\boldsymbol{e}$ indicate the focal projection from areas $24 \mathrm{~b}, 14$, and 32 , respectively. Note the converging and interdigitation of the labeled clusters. The white arrowheads indicate the correspondent blood vessels in adjacent sections.

tions (Fig. 3a). The focal projections from the dACC and OFC regions did not occupy completely separate territories in any part of the striatum (Fig. $3 b-j$ ) but converged most extensively at rostral levels. In contrast, there was greater separation of projections between terminals from the vmPFC and the dACC/OFC, particularly at caudal levels (Fig. 3i,j). Unlike the concentration of terminal fields of the vmPFC, dACC, and OFC located anterior to the anterior commissure, those from the DLPFC occupied a longitudinal dorsal and central strip from the rostral pole of the striatum, extending caudally. Patches of dense DLPFC terminal fields were also located in the putamen adjacent to the internal capsule. At rostral levels, DLPFC terminals converged with those from both the dACC and OFC (Fig. $3 e-j$ ), although each cortical projection also occupied its own territory. Here, projections from all PFC areas occupied a central region, with each cortical projection extending into nonoverlapping zones. Convergence was less prominent caudally, with almost complete separation of the dense terminals from the DLPFC and dACC/OFC just rostral to the anterior commissure (Fig. 3j).

Several individual cases that had two or more tracer injections in different cortical regions (Fig. 4) illustrate the convergence between focal projection fields and interdigitation at the borders of those projections. Case 124 (Fig. $4 a, b)$ had an injection into $\mathrm{dACC}$ (area 24b) and one into OFC (area 13). Case 133 (Fig. 4e,f) had an injection into dACC (area 32) and OFC (area 11). In both cases, portions of the focal projections converged in the central and medial rostral striatum. Case 115 (Fig. 4c,d) had an injection into the vmPFC and one into the OFC (area 13). Focal projections fields from these two injection sites converged rostral to the nucleus accumbens. Figure 4, $a, c$, and $e$, shows the outline of the focal projection (from areas $24 \mathrm{~b}$, vmPFC, and 32 , respectively) superimposed on its dense terminal field. Figure $4, b, d$, and $f$, shows that outline placed on the adjacent section that was processed for the second tracer placed in respective areas 13, 13, and 11 .

\section{Distribution of diffuse terminal fields}

Each injection produced many labeled fibers that extended outside of the focal terminal fields that were used for the 3-D reconstructions (Fig. 2). We compiled these charted fibers from each case into one map to illustrate the extent of the diffuse projection areas from the vmPFC, OFC, dACC, and DLPFC (Fig. 5). Fiber clusters surrounded and increased the area of the focal projections. In addition, they extended some distances from them, invading striatal regions that receive their focal input from other prefrontal cortex areas. To demonstrate the convergence between the diffuse terminals fields and focal projections from different cortical regions, we also illustrate the collective chartings superimposed onto the focal projection field maps (Fig. $5 g-j$ ). The focal projection from vmPFC was relatively confined to the nucleus accumbens and medial caudate nucleus. Clusters of terminals were also located in more dorsal and lateral striatal regions (Fig. 5a,b) (see also Fig. 2a), within the distal focal projections fields from the OFC, and dACC, and the diffuse projections from the DLPFC (Fig. $5 c-f$ ). However, it is of particular interest that the diffuse projections from the vmPFC remained limited to the immediate area surrounding its focal projection, thus confining both the diffuse and focal projections from the vmPFC projections to a relatively small and limited ventromedial striatal position.

The diffuse projections from dACC extended its projection field in all directions (Fig. $5 a, b$ ) (see also Fig. $2 b$ ), overlapping with the focal projections from the vmPFC, OFC, and DLPFC (Fig. $5 g-i$ ). Unlike terminals from the vmPFC, clusters of fibers were found in the dorsal lateral caudate nucleus and in the caudal ventral putamen, areas that did not receive a focal input from other prefrontal regions studied (Fig. $5 b, c$ ). They were also scattered in the medial half of the putamen, caudal to the anterior commissure, an area that also contains few fibers from other prefrontal regions (Fig. 5i,j). Together, fibers from the dACC invaded almost the entire rostral striatum, with extensive convergence with projections from both the OFC and the DLPFC (Fig. $5 d-j$.). The diffuse projection from the OFC expanded its focal projection into the rostral pole (Fig. 5a) (see also Fig. $2 c$ ) and into the dorsal and central caudate and putamen. Like the dACC, the OFC innervated a large portion of the rostral striatum with extensive convergence with the focal and diffuse projections from both the dACC and the DLPFC (Fig. $5 c-h$ ). However, at more caudal levels, there were few OFC fibers in the dorsolateral region. Here the diffuse projection was concentrated in the dorsal caudate and ventral putamen (Fig. $5 f, i, j$ ).

Clusters of DLPFC fibers terminated throughout the rostral striatum, including the ventral striatum and lateral putamen (Fig. $5 d-f$ ) (see also Fig. $2 d$ ). The combination of focal and diffuse projections demonstrates that the DLPFC occupied much of the 

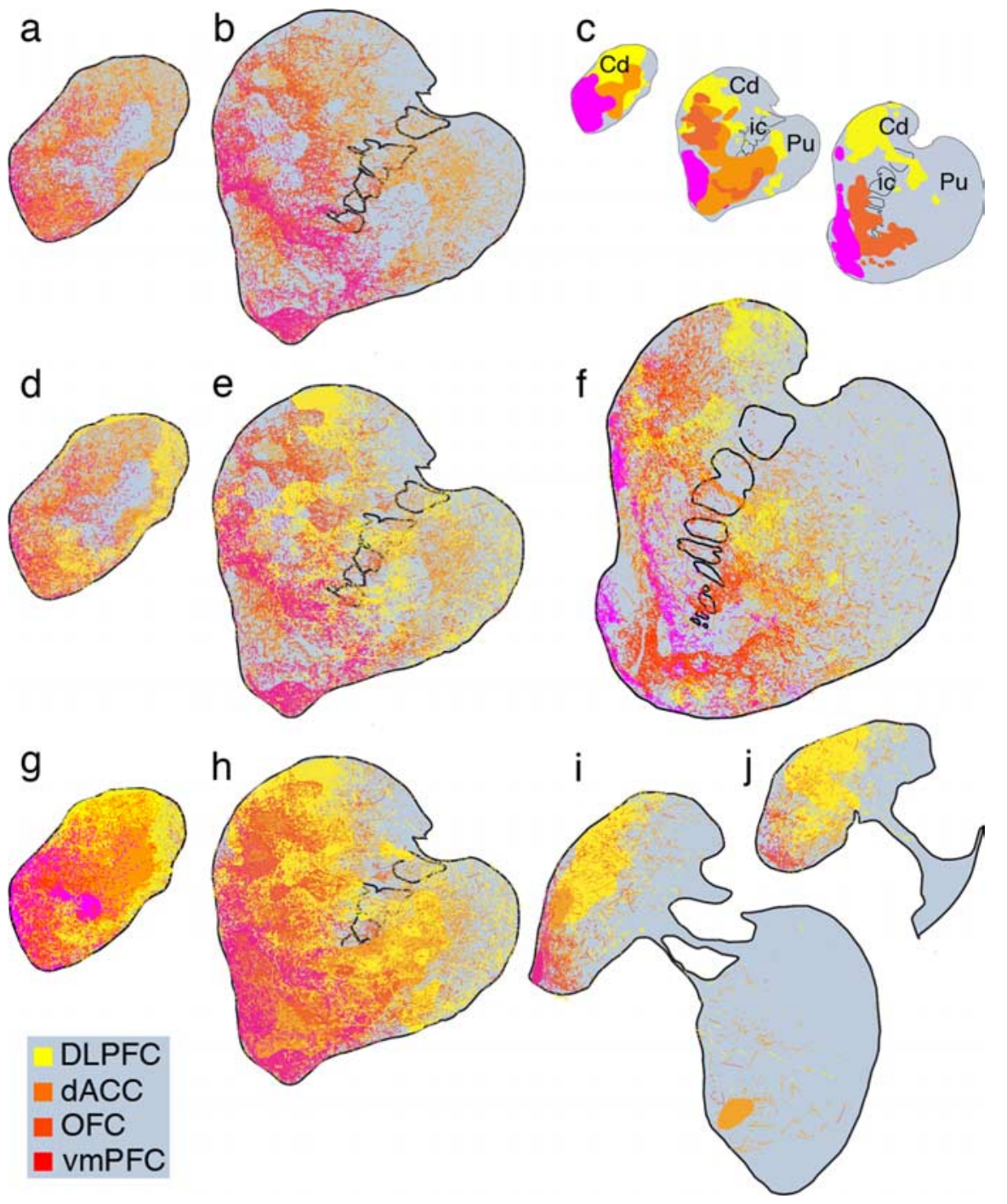

Figure 5. Charts of labeled terminals at different anteroposterior levels of the striatum. $\boldsymbol{a}, \boldsymbol{b}$, Collective diffuse projections from the ACC and OFC. $\boldsymbol{c}$, The focal projection fields are represented as colored areas in the small schematics; $\boldsymbol{d}-\boldsymbol{f}$, interface between ACC/OFC diffuse projections and those from the DLPFC; $\boldsymbol{g}-\boldsymbol{j}$, diffuse projections are superimposed onto the dense projection showing the interface of the diffuse and focal projections. Cd, Caudate nucleus; ic, internal capsule; Pu, putamen nucleus.

rostral striatum and continued caudally through the caudate nucleus Although the focal projections did not reach into the ventromedial region, clusters of labeled fibers were located here after all injections into the DLPFC. The extensive PFC-striatal projections from each limited injection site placed into specific areas is illustrated in a case in which a small, confined PHA-L injection was placed into area $24 \mathrm{a}$. The tracer was taken up by few cells, allowing the tracing of individual axons. In the same case, a larger injection of LY was placed into area 9, resulting in the typical dense patchy terminal distribution. Figure 6 illustrates two single PHA-L-positive fibers invading the densely innervated territory of area 9 projections.

\section{Discussion}

\section{The reward-related striatum}

The nucleus accumbens is a relatively small area occupying the ventral, medial striatum. The ventral striatum is larger and includes the nucleus accumbens and adjacent caudate nucleus and putamen (Haber and McFarland, 1999). The reward-related striatum as defined by ACC/OFC inputs from our injection sites occupies at least $22 \%$ of the striatum. Furthermore, the reward-related striatum is not limited to the ventral striatum but extends into a large medial and central region of the dorsal striatum and occupies much of the rostral pole before tapering off caudally. The large extent of this region is consistent with the findings that diverse striatal areas are activated after rewardrelated behavioral paradigms (Takikawa et al., 2002; Corlett et al., 2004; Haruno et al., 2004; Kawagoe et al., 2004; Tanaka et al., 2004; Tricomi et al., 2004; Kuhnen and Knutson, 2005). There are regions of convergence between the focal terminal fields that provide an anatomical substrate for integration between different rewardprocessing circuits within specific striatal areas. Because medium spiny neurons have a dendritic field spanning up to 0.5 $\mathrm{mm}$ in all directions (Wilson, 2004), the area of convergence is likely to be larger than estimated based solely on the relationship between the afferent projections alone. Areas of converging terminals may represent "hot spots" of plasticity for integrating reward value, predictability, and salience and may be especially vulnerable to the development of addictive disorders.

Previous studies show that corticostriatal terminals from the functionally related sensorimotor system converge at their interface in the striatum (Flaherty and Graybiel, 1993; Takada et al., 1998). Here, axons from each area synapse onto single fast-spiking GABAergic interneurons. These cells are more responsive to cortical input than the medium spiny cells (Charpier et al., 1999; Ramanathan et al., 2002; Mallet et al., 2005). Our data demonstrate that reward-processing information from different cortical areas follow a similar pattern of convergence. The complexity of these interactions is consistent with the concept that integrative aspects of striatal processing are organized around the striatal compartmentation of cortical inputs (Graybiel and Penney, 1999). Behavioral guiding rules are developed from different aspects of reward-related information, including reward value, expectation, and predictability. Regions of converging inputs may be particularly critical for the coordination of these processes.

\section{Interactions between the focal prefrontal corticostriatal projections}

In addition to convergence between vmPFC, dACC, and OFC focal terminals, projections from $\mathrm{dACC}$ and OFC also converge with inputs from the DLPFC, demonstrating that functionally diverse PFC projections also converge in the striatum. This is particularly prominent rostral to the level conventionally defined as the nucleus accumbens. Coordinated activation of DLPFC, dACC, and/or OFC terminals in the striatum would produce a unique combinatorial activation at the specific sites sensitive to learning. Convergence of these pathways implies a central role for 

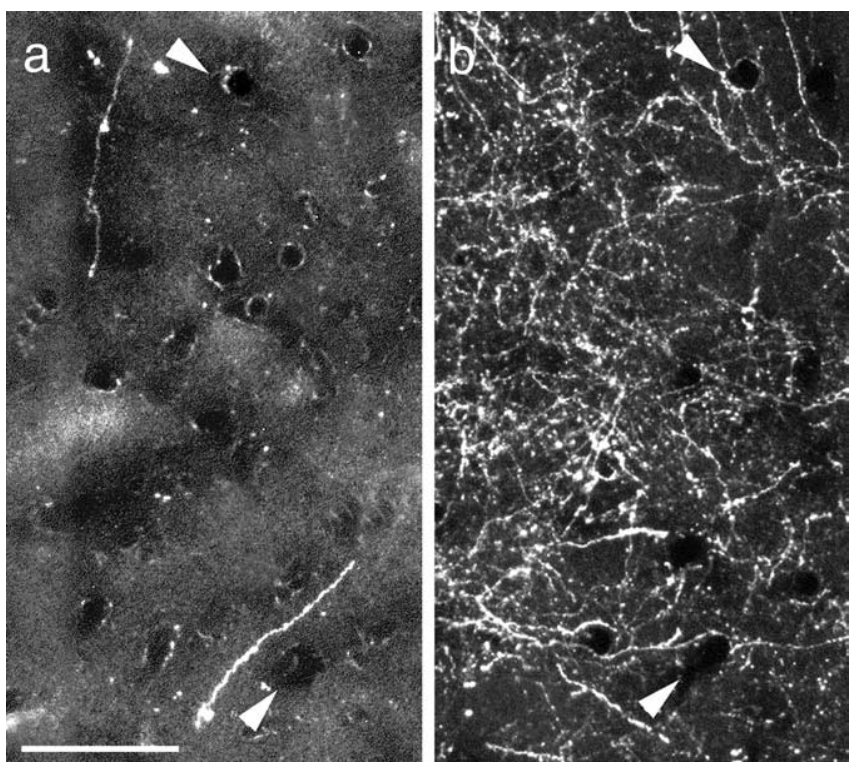

Figure 6. After a small PHA-L injection into area $24 a$, single fibers are found in the dorsal striatum (a), in the area that receives its focal input from area $9 \mathrm{~m}(\boldsymbol{b})$. Scale bar, $100 \mu \mathrm{m}$.

the rostral and dorsal striatal areas to synchronize different aspects of reward and learning, making it a unique region for reward-based incentive drive to impact on long-term strategic planning and habit formation (Everitt and Robbins, 2005).

\section{The role of the diffuse projections}

There is a significant and extensive diffuse projection from each PFC region that further increases the expanse of influence for each area. Clusters of fibers from all PFC areas invade other functional regions at some distance, a finding that is consistent with the demonstration that a single corticostriatal axon can innervate $14 \%$ of the striatum (Zheng and Wilson, 2002). However, activation of medium spiny neuron requires a large coordinated glutamatergic input from many cortical cells (Wilson, 2004). Therefore, the invasions of relatively small fiber clusters from other functional regions are not considered to have much relevance for corticostriatal information processing, and, as a result, anatomical studies have focused on the large, dense focal projections (Selemon and Goldman-Rakic, 1985; Alexander et al., 1990; Ferry et al., 2000). Indeed, under normal conditions in which a routine behavior is executed, these fibers may have little impact. However, clusters of fibers that penetrate into other functional regions at some distance may serve a separate integrative function from those that converge at the boundaries of the focal projection fields. Together, diffuse projections from diverse prefrontal cortex represent a large population of axons invading each focal projection field. Under certain conditions, if these axons were collectively activated, they might provide the recruitment strength necessary to modulate and potentially change the incoming focal signal. Finally, although research emphasis has been placed on cortical input to the striatum, there is also a massive glutamatergic thalamic input, which differs in its specific synaptic targets (Smith et al., 2004). Clusters of cortical axons that invade other functional territories may interact more specifically with thalamic glutamatergic input, providing a small but potentially significant functionally diverse signal. Although a specific role for the diffuse projections is unclear, it may serve to broadly disseminate cortical activity to a wide striatal region.

\section{The striatum and incentive learning}

Previous studies emphasize separate terminal fields from medial and orbital prefrontal cortex networks in the striatum (Haber et al., 1995; Ferry et al., 2000), in line with the concept that corticostriatal pathways are organized in functionally segregated, parallel pathways, with no substantial overlap between cortical loops (Alexander et al., 1990). The present results show an organization that features a dual system permitting both parallel and integrative network processing. Parallel processing provides throughput channels by which specific actions can be expressed but others are inhibited, allowing selected behaviors without interference from unwanted ones (Mink, 1996; Hikosaka et al., 1999). However, the role of the basal ganglia in learning new procedures and associations suggests the necessity for integrative processing across circuits (Corlett et al., 2004; Haruno et al., 2004; Tricomi et al., 2004). The hot spots of interface between fibers from different prefrontal cortex regions may be the zone for dynamic restructuring of neural ensembles fundamental to learning and habit formation. Indeed, changes in striatal activation during learning can occur in the striatum before cortex (Pasupathy and Miller, 2005). In this regard, it is of particular interest that the dACC and DLPFC innervate striatal regions outside the focal PFC projection fields, in premotor and motor regions, areas important for planning actions.

Animal and human studies emphasize the essential role of the striatum in processing reward-related information. However, precisely what that role is remains in debate. The relevant signal for reward prediction error or reward saliency that mediates the striatal incentive-related learning process is thought to originate in the midbrain DA cells and conveyed to all striatal circuits (Pagnoni et al., 2002; Schultz, 2002; Satoh et al., 2003). The latency between the presentation of the reward stimuli and the activity of the DA cells is too short to reflect higher cortical processing necessary for linking a stimulus with its rewarding properties. The fast, burst-firing activity is likely, therefore, to be generated from other input such as in the brainstem glutamatergic nuclei (Dommett et al., 2005). However, a critical issue is, how do the dopamine cells receive information concerning reward value? The largest forebrain input to the dopamine neurons is from the striatum, and the largest input to the striatum is from cortex (for review, see Haber and Gdowski, 2004). Collectively, the prefrontal cortical inputs to the striatum are in a position to modulate striatal response to different aspects of reward saliency. In addition, parts of the striatal area that receive the ACC/OFC inputs also receive inputs from the amygdala and the hippocampus, regions associated with emotional valence and memory, respectively (Friedman et al., 2002; Fudge et al., 2002). Together, these connections integrate information related to reward processing and memory to modulate striatal activity (O'Donnell and Grace, 1995). This complex network of PFC, amygdala, and hippocampal inputs processing salient stimuli impacts directly on a subset of medial dopamine neurons, which, through a series of connections, influences the dorsal striatum (Haber et al., 2000). Thus, although the short-latency burst firing activity of dopamine that signals the immediate reinforcement is likely to be triggered from brainstem nuclei, the cortico-striato-midbrain pathway is in the position to train dopamine cells to distinguish rewards and calculate error prediction and to change over time to impact on the dorsal striatum (Everitt and Robbins, 2005). This pathway may then be reinforced via the nigrostriatal pathway, placing the striato-nigro-striatal pathway in a pivotal position for learning (Haber et al., 2000; Everitt and Robbins, 2005). 
In summary, the corticostriatal network constitutes a system in which prefrontal cortex exploits the basal ganglia for additional processing of reward and cognition to effectively modulate learning and decision making that leads to the development of goal-directed behaviors and habit formation. Furthermore, the rostralmost part of the striatum is a unique region for integrating these functions, an area likely to be larger in humans than in nonhuman primates given the evolutionary expansion of prefrontal cortex.

\section{References}

Alexander GE, Crutcher MD, DeLong MR (1990) Basal gangliathalamocortical circuits: parallel substrates for motor, oculomotor, "prefrontal" and "limbic" functions. Prog Brain Res 85:119-146.

Barbas H, Pandya DN (1989) Architecture and intrinsic connections of the prefrontal cortex in the rhesus monkey. J Comp Neurol 286:353-375.

Carmichael ST, Price JL (1996) Connectional networks within the orbital and medial prefrontal cortex of macaque monkeys. J Comp Neurol 371:179-207.

Charpier S, Mahon S, Deniau JM (1999) In vivo induction of striatal longterm potentiation by low-frequency stimulation of the cerebral cortex. Neuroscience 91:1209-1222.

Corlett PR, Aitken MR, Dickinson A, Shanks DR, Honey GD, Honey RA, Robbins TW, Bullmore ET, Fletcher PC (2004) Prediction error during retrospective revaluation of causal associations in humans: fMRI evidence in favor of an associative model of learning. Neuron 44:877-888.

Dommett E, Coizet V, Blaha CD, Martindale J, Lefebvre V, Walton N, Mayhew JE, Overton PG, Redgrave P (2005) How visual stimuli activate dopaminergic neurons at short latency. Science 307:1476-1479.

Elliott R, Dolan RJ, Frith CD (2000a) Dissociable functions in the medial and lateral orbitofrontal cortex: evidence from human neuroimaging studies. Cereb Cortex 10:308-317.

Elliott R, Friston KJ, Dolan RJ (2000b) Dissociable neural responses in human reward systems. J Neurosci 20:6159-6165.

Elliott R, Newman JL, Longe OA, Deakin JF (2003) Differential response patterns in the striatum and orbitofrontal cortex to financial reward in humans: a parametric functional magnetic resonance imaging study. J Neurosci 23:303-307.

Everitt BJ, Robbins TW (2005) Neural systems of reinforcement for drug addiction: from actions to habits to compulsion. Nat Neurosci 8:1481-1489.

Fellows LK, Farah MJ (2005) Different underlying impairments in decisionmaking following ventromedial and dorsolateral frontal lobe damage in humans. Cereb Cortex 15:58-63.

Ferry AT, Ongur D, An X, Price JL (2000) Prefrontal cortical projections to the striatum in macaque monkeys: evidence for an organization related to prefrontal networks. J Comp Neurol 425:447-470.

Flaherty AW, Graybiel AM (1993) Two input systems for body representations in the primate striatal matrix: experimental evidence in the squirrel monkey. J Neurosci 13:1120-1137.

Friedman DP, Aggleton JP, Saunders RC (2002) Comparison of hippocampal, amygdala, and perirhinal projections to the nucleus accumbens: combined anterograde and retrograde tracing study in the macaque brain. J Comp Neurol 450:345-365.

Fudge JL, Kunishio K, Walsh C, Richard D, Haber SN (2002) Amygdaloid projections to ventromedial striatal subterritories in the primate. Neuroscience 110:257-275.

Goldman-Rakic PS, Funahashi S, Bruce CJ (1990) Neocortical memory circuits. Cold Spring Harb Symp Quant Biol 55:1025-1038.

Graybiel AM, Penney JB (1999) Chemical achitecture of the basal ganglia. In: Handbook of chemical neuroanatomy (Bloom FE, Bjorklund A, Hokfelt T, eds), pp 227-284. New York: Elsevier Science.

Haber SN, Gdowski MJ (2004) The basal ganglia. In: The human nervous system, Ed 2 (Paxinos G, Mai JK, eds), pp 676-738. New York: Elsevier Academic.

Haber SN, McFarland NR (1999) The concept of the ventral striatum in nonhuman primates. In: Advancing from the ventral striatum to the extended amygdala (McGinty JF, ed), pp 33-48. New York: New York Academy of Sciences.
Haber SN, Lynd-Balta E, Mitchell SJ (1993) The organization of the descending ventral pallidal projections in the monkey. J Comp Neurol 329:111-129.

Haber SN, Kunishio K, Mizobuchi M, Lynd-Balta E (1995) The orbital and medial prefrontal circuit through the primate basal ganglia. J Neurosci 15:4851-4867.

Haber SN, Fudge JL, McFarland NR (2000) Striatonigrostriatal pathways in primates form an ascending spiral from the shell to the dorsolateral striatum. J Neurosci 20:2369-2382.

Hadland KA, Rushworth MF, Gaffan D, Passingham RE (2003) The anterior cingulate and reward-guided selection of actions. J Neurophysiol 89:1161-1164.

Haruno M, Kuroda T, Doya K, Toyama K, Kimura M, Samejima K, Imamizu H, Kawato M (2004) A neural correlate of reward-based behavioral learning in caudate nucleus: a functional magnetic resonance imaging study of a stochastic decision task. J Neurosci 24:1660-1665.

Hassani OK, Cromwell HC, Schultz W (2001) Influence of expectation of different rewards on behavior-related neuronal activity in the striatum. J Neurophysiol 85:2477-2489.

Hikosaka O, Nakahara H, Rand MK, Sakai K, Lu X, Nakamura K, Miyachi S, Doya K (1999) Parallel neural networks for learning sequential procedures. Trends Neurosci [Erratum (1999) 22:after 568] 22:464-471.

Kawagoe R, Takikawa Y, Hikosaka O (2004) Reward-predicting activity of dopamine and caudate neurons-a possible mechanism of motivational control of saccadic eye movement. J Neurophysiol 91:1013-1024.

Knutson B, Adams CM, Fong GW, Hommer D (2001) Anticipation of increasing monetary reward selectively recruits nucleus accumbens. J Neurosci 21:RC159(1-5)

Kremer JR, Mastronarde DN, McIntosh JR (1996) Computer visualization of three-dimensional image data using IMOD. J Struct Biol 116:71-76.

Kuhnen CM, Knutson B (2005) The neural basis of financial risk taking. Neuron 47:763-770.

Kunishio K, Haber SN (1994) Primate cingulostriatal projection: limbic striatal versus sensorimotor striatal input. J Comp Neurol 350:337-356.

Mallet N, Le Moine C, Charpier S, Gonon F (2005) Feedforward inhibition of projection neurons by fast-spiking GABA interneurons in the rat striatum in vivo. J Neurosci 25:3857-3869.

Mink JW (1996) The basal ganglia: focused selection and inhibition of competing motor programs. Prog Neurobiol 50:381-425.

National Research Council (1996) Guide for the care and use of laboratory animals. Washington, DC: National Academy.

O'Donnell P, Grace AA (1995) Synaptic interactions among excitatory afferents to nucleus accumbens neurons: hippocampal gating of prefrontal cortical input. J Neurosci 15:3622-3639.

Pagnoni G, Zink CF, Montague PR, Berns GS (2002) Activity in human ventral striatum locked to errors of reward prediction. Nat Neurosci 5:97-98.

Parthasarathy HB, Schall JD, Graybiel AM (1992) Distributed but convergent ordering of corticostriatal projections: analysis of the frontal eye field and the supplementary eye field in the macaque monkey. J Neurosci 12:4468-4488.

Pasupathy A, Miller EK (2005) Different time courses of learning-related activity in the prefrontal cortex and striatum. Nature 433:873-876.

Paxinos G, Huang XF, Toga AW (2000) The rhesus monkey in stereotaxic coordinates. San Diego: Academic.

Ramanathan S, Hanley JJ, Deniau JM, Bolam JP (2002) Synaptic convergence of motor and somatosensory cortical afferents onto GABAergic interneurons in the rat striatum. J Neurosci 22:8158-8169.

Satoh T, Nakai S, Sato T, Kimura M (2003) Correlated coding of motivation and outcome of decision by dopamine neurons. J Neurosci 23:9913-9923.

Schultz W (2002) Getting formal with dopamine and reward. Neuron 36:241-263.

Schultz W, Tremblay L, Hollerman JR (2000) Reward processing in primate orbitofrontal cortex and basal ganglia. Cereb Cortex 10:272-284.

Selemon LD, Goldman-Rakic PS (1985) Longitudinal topography and interdigitation of corticostriatal projections in the rhesus monkey. J Neurosci 5:776-794.

Smith Y, Raju DV, Pare JF, Sidibe M (2004) The thalamostriatal system: a highly specific network of the basal ganglia circuitry. Trends Neurosci 27:520-527. 
Takada M, Tokuno H, Nambu A, Inase M (1998) Corticostriatal input zones from the supplementary motor area overlap those from the contrarather than ipsilateral primary motor cortex. Brain Res 791:335-340.

Takikawa Y, Kawagoe R, Hikosaka O (2002) Reward-dependent spatial selectivity of anticipatory activity in monkey caudate neurons. J Neurophysiol 87:508-515.

Tanaka SC, Doya K, Okada G, Ueda K, Okamoto Y, Yamawaki S (2004) Prediction of immediate and future rewards differentially recruits cortico-basal ganglia loops. Nat Neurosci 7:887-893.

Tricomi EM, Delgado MR, Fiez JA (2004) Modulation of caudate activity by action contingency. Neuron 41:281-292.

Volkow ND, Wang GJ, Ma Y, Fowler JS, Wong C, Ding YS, Hitzemann R, Swanson JM, Kalivas P (2005) Activation of orbital and medial prefron- tal cortex by methylphenidate in cocaine-addicted subjects but not in controls: relevance to addiction. J Neurosci 25:3932-3939.

Walton ME, Devlin JT, Rushworth MF (2004) Interactions between decision making and performance monitoring within prefrontal cortex. Nat Neurosci 7:1259-1265.

Wilson CJ (2004) The basal ganglia. In: Synaptic organization of the brain, Ed 5 (Shepherd GM, ed), pp 361-413. New York: Oxford UP.

Yeterian EH, Van Hoesen GW (1978) Cortico-striate projections in the rhesus monkey: the organization of certain cortico-caudate connections. Brain Res 139:43-63.

Zheng T, Wilson CJ (2002) Corticostriatal combinatorics: the implications of corticostriatal axonal arborizations. J Neurophysiol 87: $1007-1017$ 